\title{
Synthesis, Characterization and Biological Evaluation of Novel 1, 4-Benzodiazepine Derivatives as Potent Anti-Tubercular Agents
}

\author{
M. Murali Krishna Kumar*, T. Mohan, G. Krupa Mai, G.P.V Sangeeta, K. Purna Nagasree \\ Pharmaceutical Chemistry Research Labs, AU College of Pharmaceutical Sciences, Andhra University, Visakhapatnam, Andhra Pradesh, INDIA.
}

\begin{abstract}
Background: Benzodiazepines are interesting compounds due to their therapeutic properties. TB chemotherapy is highly regulated and Its research research is often considered non-remunerative. Hence, there remains a pressing requisite to discover novel, potent and safe anti-TB agents. Objective: One of the biggest problem of Tuberculosis is the lack of effective treatments. Bedaquiline (2013) and Delaminid (2014) are the only two agents approved for treating tuberculosis after Rifampicin (1963). This clearly shows the need for new lead molecules to fight against tuberculosis. Methodology: In the present work, a series of eighteen derivatives of 1,4-Benzodiazepines were synthesized by condensation of o-phenylenediamines with 1, 3-diketone (Dimedone). Further these synthesized derivatives were analyzed by IR, NMR and MASS spectral studies and are screened for anti-tubercular and antimicrobial activity. Results: Among these, potent activity were observed for compounds 9, 10 (MIC $1.6 \mu \mathrm{g} / \mathrm{mL}$ ) followed by $11(3.12 \mu \mathrm{g} / \mathrm{mL}), 04(6.25 \mu \mathrm{g} / \mathrm{mL})$ against $M$. tuberculosis
\end{abstract}

H37Rv. We report here the synthesis, screening data and SAR studies of Benzodiazepines and its derivatives as Antitubercular agents. Conclusion: In conclusion, we synthesized eighteen 1, 4-Benzodiazepines derivatives with selective anti-tubercular activity.

Key words: Benzodiazepines, M. tuberculosis H37Rv, Antitubercular. Key message: We report here a new method for the preparation of some novel 1, 4-benzodiazepine derivatives with selective anti TB activity.

Correspondence

Dr. M. Murali Krishna Kumar, Pharmaceutical Chemistry Research Labs, AU College of Pharmaceutical Sciences, Andhra University, Visakhapatnam - 530 003, Andhra Pradesh, INDIA.

Phone: 9441419246

Email: drmmkau@gmail.com

DOI: 10.5530/jyp.2018.10.60

\section{INTRODUCTION}

Tuberculosis is one of the oldest infectious disease known to mankind. It is known for Its ease of spread, lengthy treatment schedule, toxicity of available drugs and drug resistance. During the period 1960-70, this disease receded significantly due to the development of effective drugs including INH, Pyrazinamide and Rifampicin. With the emergence of multi drug resistance (MDR) and extremely drug resistance (XDR) TB in the last 15 years, TB regained as global emergency status. ${ }^{1}$ It is estimated that in 2014-15 alone, $3.3 \%$ of new TB cases and $20 \%$ of previously treated cases were found to have MDR-TB and around 1,90,000 people died of MDR-TB. Extensively drug-resistant TB (XDR-TB) had been reported by 105 countries by 2015 . An estimated $9.7 \%$ of people with MDR-TB have XDR-TB. Treatment of MDRTB/XDR TB complicates the treatment protocol due to scarcity of effective drugs, drug toxicity and patient non-compliance..$^{2-4}$ Bedaquiline (in 2013) and delamanid (in 2014) are the only two novel drugs approved for treatment of MDRTB. ${ }^{5}$ Regulation of TB Chemotherapy is highly regulated and anti TB drug research is often considered non-remunerative. Hence, there remains a pressing requisite to discover novel, potent and safe anti-TB agents.

Benzodiazepines are interesting compounds because of their therapeutic properties. ${ }^{6}$ Many derivatives of this family are nowadays widely used as tranquilizing and anticonvulsant agents. Though the first benzodiazepine introduced as a drug 30 years ago, ${ }^{7}$ the research in this area is still very active and directed towards the synthesis of compounds with enhanced pharmacological activity. Benzodiazepines are useful in treating anxiety, anti-convulsant, sedative, anti-depressant, ${ }^{8}$ platelet-activating factor antagonists, HIV trans-activator, ${ }^{9} \mathrm{HIV}$ reverse transcriptase inhibitors, ${ }^{10}$ insomnia, seizures, muscle relaxant properties, effect on the neurotransmitter, alcohol withdrawal, gamma-aminobutyric acid (GABA-A) ${ }^{11}$ and anti-tumor. ${ }^{12}$

Despite of their wide range of pharmacological activities, industrial and synthetic applications, the synthesis of benzodiazepines has received little attention. The literature methods for the synthesis of benzodiazepines which have been reported recently, include condensation reactions of o-phenylenediamines with unsaturated carbonyl compounds, ${ }^{13}$ haloketones, ${ }^{14}$ or with ketones in the presence of catalysts like BF 3 -etherate, ${ }^{15}$ $\mathrm{NaBH}_{4},{ }^{16}$ polyphosphoric acid or $\mathrm{SiO}_{2},{ }^{17}$ Sodium tetrachloroaurate (III) dehydrate, ${ }^{18}$ boron solfunic acid ${ }^{19-25}$ under solvent conditions and ionic Liquid. ${ }^{26}$

We report here a new method for the preparation of 1, 4-benzodiazepine derivatives by condensation of o-phenylenediamines with 1, 3-diketone (Dimedone).

\section{MATERIALS}

All the chemicals were purchased from Aldrich Chemical Company (USA) and were used without further purification. The reactions were monitored by pre-coated aluminum silica gel TLC plates. Iodine vapors are used as visualizing agents. Melting points (m.p) were determined using an SRS-EZ Melt automated melting point instrument, without correction. The IR spectra were recorded on BRUKER FT-IR (software - OPUS 6.4) spectrometer using $\mathrm{KBr}$ disc method and the values were expressed in $\mathrm{cm}^{-1}$. The ${ }^{1} \mathrm{H}-\mathrm{NMR}$ spectra of the compounds were recorded in DMSO-d6 or $\mathrm{CDCl}_{3}$ with BRUKER AVANCE $400 \mathrm{MHz}$ NMR spectrometer (software - Topspin 3.2) and chemical shifts were expressed in $\delta(\mathrm{ppm})$. Shifts reported are relative to the signal of the solvent used in each case and coupling constants are reported in $\mathrm{Hz}$ (s: singlet, bs: broad 


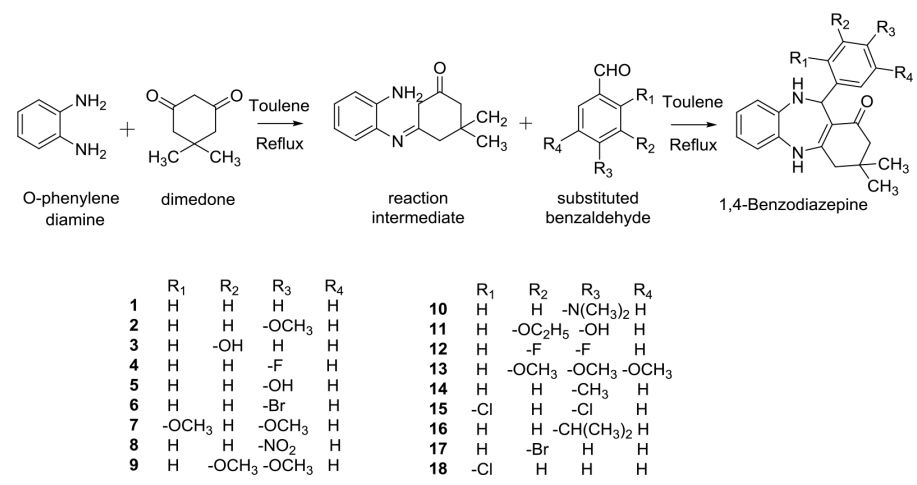

SCHEME-I: Synthesis of 1, 4-benzodiazepine derivatives 1-18

singlet, d: doublet, t: triplet, dd: double doublet, dt: double triplet, $\mathrm{m}$ : multiplet). The mass spectra were recorded on AGILENT QQQ LC-MS (ESI-MS) spectrometer (software - Mass Hunter B.03.01).

\section{METHODS}

\section{Synthesis of 1, 4-Benzodiazepines from various} aldehydes:

o-Phenylene diamine $(1 \mathrm{~mol})$, Dimedone $(1 \mathrm{~mol})$ were weighed and placed in round bottomed flask. Then toluene was added and refluxed $\left(1^{1 / 2 h}\right)$ by using Dean stark apparatus. Later the reaction mixture was allowed to attain room temperature. During cooling semi solid intermediate settles down in the RBF. Then the solvent was decanted. To it, aldehyde (1mol) was added along with fresh toluene $(10 \mathrm{ml})$ and refluxed $\left(1^{1 / 2 h}\right)$. After reflux, the reaction mixture was monitored by the TLC using hexane and ethyl acetate as mobile phase. The reaction mixture was filtered and washed with diethyl ether to obtain pure product.

\section{Characterization of synthesized compounds}

1-((E)-2,5-dihydro-2-phenyl-1H-benzo[b] [1,4]diazepin)-5-dimethylcyclohexanone (1): It was obtained as a orange solid; yield $54.15 \%$; m.p 241.1 ${ }^{\circ} \mathrm{C}$; Anal. calc for $\mathrm{C}_{21} \mathrm{H}_{22} \mathrm{~N}_{2} \mathrm{O}$ C, $79.21 ; \mathrm{H}, 6.96 ; \mathrm{N}, 8.80$; found: $\mathrm{C}$, 72.19; H, 6. 94; N, 8.78; IR (KBr) v max: $\mathrm{cm}^{-1}$ : 3306, 3184, 2954, 1580, 1510 and $1386 \mathrm{~cm}^{-1} ;{ }^{1} \mathrm{H}$ NMR $\left(\mathrm{CDCl}_{3}, 400 \mathrm{MHz}\right) ; 1.11\left(3 \mathrm{H}, \mathrm{s}, \mathrm{CH}_{3}\right) ; 1.18$ $\left(3 \mathrm{H}, \mathrm{s}, \mathrm{CH}_{3}\right) ; 2.24\left(1 \mathrm{H}, \mathrm{d}, \mathrm{J}=16.4, \mathrm{CH}_{2}\right) ; 2.33\left(1 \mathrm{H}, \mathrm{d}, \mathrm{J}=16, \mathrm{CH}_{2}\right) ; 2.42(1 \mathrm{H}$, d, J=15.6, $\left.\mathrm{CH}_{2}\right) ; 2.62\left(1 \mathrm{H}, \mathrm{d}, \mathrm{J}=15.6, \mathrm{CH}_{2}\right) ; 5.95(1 \mathrm{H}, \mathrm{s}, \mathrm{CH}) ; 6.38(4 \mathrm{H}, \mathrm{m}$, Ar-H); 7.07 (5H, m, Ar-H); ESI MS m/z: 319[M+1]

1-(E)-2,5-dihydro-2-(4-methoxyphenyl)-1H-benzo[b][1,4]diazepin)5-dimethylcyclohexanone (2): It was obtained as a orange solid. Yield $65.46 \%$. m.p $161.5^{\circ} \mathrm{C}$; Anal. calc for $\mathrm{C}_{22} \mathrm{H}_{24} \mathrm{~N}_{2} \mathrm{O}_{2:} \mathrm{C}, 75.83 ; \mathrm{H}, 6.94 ; \mathrm{N}$, 8.04; found: C, 75.82; H, 6.92; N, 8.01; IR (KBr) v max: $\mathrm{cm}^{-1}: 3321,3175$, 2926, 1578, 1504, 1381 and $1122 \mathrm{~cm}^{-1} ;{ }^{1} \mathrm{H} \mathrm{NMR}\left(\mathrm{CDCl}_{3}, 400 \mathrm{MHz},\right): 1.10$ $\left(3 \mathrm{H}, \mathrm{s}, \mathrm{CH}_{3}\right) ; 1.17\left(3 \mathrm{H}, \mathrm{s}, \mathrm{CH}_{3}\right) ; 2.23\left(2 \mathrm{H}, \mathrm{s}, \mathrm{CH}_{2}\right) ; 2.27\left(2 \mathrm{H}, \mathrm{s}, \mathrm{CH}_{2}\right) ; 2.38$ $\left(3 \mathrm{H}, \mathrm{q}, \mathrm{OCH}_{3}\right) ; 2.61-2.64(2 \mathrm{H}, \mathrm{d}, \mathrm{J}=12, \mathrm{NH}) ; 5.91(1 \mathrm{H}, \mathrm{s}, \mathrm{CH}) ; 6.44-67.02$ $(8 \mathrm{H}, \mathrm{m}, \mathrm{Ar}-\mathrm{H}) ; \mathrm{ESI} \mathrm{MS} \mathrm{m} / \mathrm{z}: 349[\mathrm{M}+1]$

((E)-2,5-dihydro-2-(3-hydroxyphenyl)-1H-benzo[b][1,4]diazepin)-5-dimethyl cyclo hexanone (3): It was obtained as a lemon yellow solid; yield 71.91\%; m.p 256.8 ${ }^{\circ} \mathrm{C}$; Anal. calc for $\mathrm{C}_{21} \mathrm{H}_{22} \mathrm{~N}_{2} \mathrm{O}_{2}$ C, 75.42; $\mathrm{H}, 6.63 ; \mathrm{N}$, 8.38; found: $\mathrm{C}, 75.40 ; \mathrm{H}, 6.62 ; \mathrm{N}, 8.37$; IR $(\mathrm{KBr}) \cup \max : \mathrm{cm}^{-1}: 3336,3240$, 2925, 1581, 1517 and $1388 \mathrm{~cm}^{-1} ;{ }^{1} \mathrm{H}$ NMR $\left(400 \mathrm{MHz}, \mathrm{CDCl}_{3}\right): 1.04(3 \mathrm{H}, \mathrm{s}$, $\left.\mathrm{CH}_{3}\right) ; 1.09\left(3 \mathrm{H}, \mathrm{s}, \mathrm{CH}_{3}\right) ; 2.05\left(2 \mathrm{H}, \mathrm{d}, \mathrm{J}=16, \mathrm{CH}_{2}\right) ; 2.18\left(2 \mathrm{H}, \mathrm{d}, \mathrm{J}=16, \mathrm{CH}_{2}\right)$; $2.57(2 \mathrm{H}, \mathrm{s}, \mathrm{NH}) ; 5.60(1 \mathrm{H}, \mathrm{d}, \mathrm{J}=4, \mathrm{CH}) ; 6.09(1 \mathrm{H}, \mathrm{d}, \mathrm{J}=4, \mathrm{OH}) ; 6.39(8 \mathrm{H}$, $\mathrm{m}, \mathrm{Ar}-\mathrm{H}) ; \mathrm{ESI} \mathrm{MS} \mathrm{m} / \mathrm{z}: 335[\mathrm{M}+1]$

((E)-2-(4-fluorophenyl)-2,5-dihydro-1H-benzo[b][1,4]diazepin)-5-dimethylcyclohexanone (4): It was obtained as a yellow solid; yield $32.55 \%$; m.p161.5 $5^{\circ}$; Anal. calc for $\mathrm{C}_{21} \mathrm{H}_{21} \mathrm{FN}_{2} \mathrm{O}$. C, 74.98; $\mathrm{H}, 6.29 ; \mathrm{N}, 8.33$; found: C, 74.96; H, 6.28; N, 8.31. IR (KBr) $v$ max: $\mathrm{cm}^{-1}: 3307,3184,2955$, 1537, 1508, 1383 and $1222 \mathrm{~cm}^{-1} ;{ }^{1} \mathrm{H}$ NMR $\left(400 \mathrm{MHz}, \mathrm{CDCl}_{3}\right): 1.10(3 \mathrm{H}$, s, $\left.\mathrm{CH}_{3}\right) ; 1.18\left(3 \mathrm{H}, \mathrm{s}, \mathrm{CH}_{3}\right) ; 2.24\left(1 \mathrm{H}, \mathrm{d}, \mathrm{J}=16, \mathrm{CH}_{2}\right) ; 2.33\left(1 \mathrm{H}, \mathrm{s}, \mathrm{CH}_{2}\right)$; $2.37\left(1 \mathrm{H}, \mathrm{d}, \mathrm{J}=4, \mathrm{CH}_{2}\right) ; 2.43\left(1 \mathrm{H}, \mathrm{d}, \mathrm{J}=12, \mathrm{CH}_{2}\right) ; 2.62(2 \mathrm{H}, \mathrm{s}, \mathrm{NH}) ; 5.93(1 \mathrm{H}$, $\mathrm{s}, \mathrm{CH}) ; 6.75(8 \mathrm{H}, \mathrm{m}, \mathrm{Ar}-\mathrm{H})$; ESI MS m/z: $337[\mathrm{M}+1]$

((E)-2,5-dihydro-2-(4-hydroxyphenyl)-1H-benzo[b][1,4]diazepin)-5-dimethyl cyclo hexanone (5): It was obtained as a yellow solid; yield $73.97 \%$; m.p 219.8 ${ }^{\circ} \mathrm{C}$; Anal. calc for $\mathrm{C}_{21} \mathrm{H}_{22} \mathrm{~N}_{2} \mathrm{O}_{2}$ C, $75.42 ; \mathrm{H}, 6.63 ; \mathrm{N}, 8.38$; found: C, 75.39; H, 6.61; N, 8.37; IR (KBr) v max: $\mathrm{cm}^{-1}: 3366,3140,2926,1580$, 1505 and $1385 \mathrm{~cm}^{-1} ;{ }^{1} \mathrm{H}$ NMR $\left(400 \mathrm{MHz}, \mathrm{CDCl}_{3}\right): 1.09\left(6 \mathrm{H}, \mathrm{m}, \mathrm{CH}_{3}\right) ; 2.20$ $\left(4 \mathrm{H}, \mathrm{m}, \mathrm{CH}_{2}\right) ; 3.13(1 \mathrm{H}, \mathrm{s}, \mathrm{NH}) ; 3.17(1 \mathrm{H}, \mathrm{s}, \mathrm{NH}) ; 3.52(1 \mathrm{H}, \mathrm{s}, \mathrm{CH}) ; 5.46$ $(1 \mathrm{H}, \mathrm{s}, \mathrm{OH}) ; 6.95(8 \mathrm{H}, \mathrm{m}, \mathrm{Ar}-\mathrm{H}) ; \mathrm{ESI} \mathrm{MS} \mathrm{m} / \mathrm{z}: 335[\mathrm{M}+1]$

((E)-2-(4-bromophenyl)-2,5-dihydro-1H-benzo[b][1,4]diazepin)-5-dimethyl cyclohexanone (6): It was obtained as a light brown solid; yield 52.01\%; m.p 220.5 ${ }^{\circ}$; Anal. calc for $\mathrm{C}_{21} \mathrm{H}_{21} \mathrm{BrN}_{2} \mathrm{O}$ C, 63.48; $\mathrm{H}, 5.33 ; \mathrm{N}$, 7.05; found:C, 63.45; H, 5.32; N, 7.03; IR (KBr) v max: $\mathrm{cm}^{-1}: 3322,3180$, 2927, 1575, 1507, 1378, and $757 \mathrm{~cm}^{-1} ;{ }^{1} \mathrm{H}$ NMR (400MHz, $\left.\mathrm{CDCl}_{3}\right): 1.07$ $\left(3 \mathrm{H}, \mathrm{s}, \mathrm{CH}_{3}\right) ; 1.14\left(3 \mathrm{H}, \mathrm{s}, \mathrm{CH}_{3}\right) ; 2.24\left(4 \mathrm{H}, \mathrm{m}, \mathrm{CH}_{2}\right) ; 2.40(1 \mathrm{H}, \mathrm{d}, \mathrm{J}=16$, $\mathrm{NH}) ; 2.55(1 \mathrm{H}, \mathrm{d}, \mathrm{J}=16, \mathrm{NH}) ; 5.90(1 \mathrm{H}, \mathrm{s}, \mathrm{CH}) ; 6.90$ (6H, m, Ar-H); 7.24 $(2 \mathrm{H}, \mathrm{m}, \mathrm{Ar}-\mathrm{H}-\mathrm{Br})$; ESI MS m/z: 397 [M-1]

(E)-2,5-dihydro-2-(2,4dimethoxyphenyl)-1H-benzo[b][1,4]diazepin)5-dimethylcyclohexanone (7): It was obtained as a white solid; yield 33.57\%; m.p-226.70 C; Anal. calc for $\mathrm{C}_{23} \mathrm{H}_{26} \mathrm{~N}_{2} \mathrm{O}_{3:} \mathrm{C}, 72.99 ; \mathrm{H}, 6.92 ; \mathrm{N}$, 7.40; found: C, 72.98; H, 6.90; N, 7.39; IR (KBr) v max: $\mathrm{cm}^{-1}: 3370,3146$, 2927, 1605, 1540, 1384 and $1130 \mathrm{~cm}^{-1},{ }^{1} \mathrm{H}$ NMR (400MHz, $\left.\mathrm{CDCl}_{3}\right): 1.16$ $\left(6 \mathrm{H}, \mathrm{s}, \mathrm{CH}_{3}\right) ; 2.25\left(2 \mathrm{H}, \mathrm{q}, \mathrm{CH}_{2}\right) ; 2.41\left(1 \mathrm{H}, \mathrm{d}, \mathrm{J}=16, \mathrm{CH}_{2}\right) ; 2.59(1 \mathrm{H}, \mathrm{d}, \mathrm{J}=16$, $\left.\mathrm{CH}_{2}\right) ; 2.27\left(2 \mathrm{H}, \mathrm{s}, \mathrm{CH}_{2}\right) ; 3.67\left(3 \mathrm{H}, \mathrm{s}, \mathrm{OCH}_{3}\right) ; 3.92\left(3 \mathrm{H}, \mathrm{s}, \mathrm{OCH}_{3}\right) ; 4.96$ $(1 \mathrm{H}, \mathrm{s}, \mathrm{CH}) ; 6.10(8 \mathrm{H}, \mathrm{m}, \mathrm{Ar}-\mathrm{H})$; ESI MS m/z: $379[\mathrm{M}+1]$

1-((E)-2,5-dihydro-2-(4-nitrophenyl-1H-benzo[b][1,4]diazepin)-5-dimethyl cyclohexanone (8): It was obtained as a yellow solid; yield 35.13\%; m.p231.8 ${ }^{\circ} \mathrm{C}$; Anal. calc for $\mathrm{C}_{21} \mathrm{H}_{21} \mathrm{~N}_{3} \mathrm{O}_{3}$. C, 69.41; H, 5.82; N, 11.56; found: C, 69.41; H, 5.82; N, 11.56; IR (KBr) v max: $\mathrm{cm}^{-1}$ : 3301, 3198, 2924, 1607, 1527, 1383 and $1276 \mathrm{~cm}^{-1}$; ${ }^{1} \mathrm{H}$ NMR $\left(400 \mathrm{MHz}, \mathrm{CDCl}_{3}\right): 1.05(3 \mathrm{H}, \mathrm{s}$, $\left.\mathrm{CH}_{3}\right) ; 1.19\left(3 \mathrm{H}, \mathrm{s}, \mathrm{CH}_{3}\right) ; 2.22\left(4 \mathrm{H}, \mathrm{m}, \mathrm{CH}_{2}\right) ; 6.00(1 \mathrm{H}, \mathrm{s}, \mathrm{CH}) ; 6.39(5 \mathrm{H}$, $\mathrm{m}, \mathrm{Ar}-\mathrm{H}) ; 7.96$ (2H, m, Ar-H-NO $)$; ESI MS m/z: 364 [M+1]

((E)-2,5-dihydro-2-(3,4-dimethoxyphenyl)-1H-benzo[b][1,4]diazepin)5-dimethylcyclohexanone (9): It was obtained as a yellow solid; yield 61.32\%; m.p 98.3 ${ }^{\circ} \mathrm{C}$; Anal. calc for $\mathrm{C}_{23} \mathrm{H}_{26} \mathrm{~N}_{2} \mathrm{O}_{3} \mathrm{C}, 72.99 ; \mathrm{H}, 6.92 ; \mathrm{N}, 7.40$; found: C, 72.97; H, 6.90; N, 7.39; IR (KBr) v max: $\mathrm{cm}^{-1}: 3307,3063,2929$, 1592, 1516, 1380 and $1143 \mathrm{~cm}^{-1},{ }^{1} \mathrm{H}$ NMR $\left(400 \mathrm{MHz}, \mathrm{CDCl}_{3}\right): 1.08(3 \mathrm{H}, \mathrm{s}$, $\left.\mathrm{CH}_{3}\right) ; 1.14\left(3 \mathrm{H}, \mathrm{s}, \mathrm{CH}_{3}\right) ; 2.2\left(4 \mathrm{H}, \mathrm{m}, \mathrm{CH}_{2}\right) ; 2.75\left(6 \mathrm{H}, \mathrm{s}, \mathrm{N}-\mathrm{CH}_{3}\right) ; 3.00(2 \mathrm{H}$, s, $\mathrm{NH}) ; 5.61(1 \mathrm{H}, \mathrm{s}, \mathrm{CH}) ; 3.66\left(3 \mathrm{H}, \mathrm{s}, \mathrm{OCH}_{3}\right) ; 3.76\left(3 \mathrm{H}, \mathrm{s}, \mathrm{OCH}_{3}\right) ; 5.89$ $(1 \mathrm{H}, \mathrm{s}, \mathrm{CH}) ; 6.44(6 \mathrm{H}, \mathrm{m}, \mathrm{Ar}-\mathrm{H}) ; \mathrm{ESI} \mathrm{MS} \mathrm{m} / \mathrm{z}: 379[\mathrm{M}+1]$

1-((E)-2-(4-dimethylamino)phenyl)-2,5dihydro-1H-benzo $[b][1,4]$ diazepin)-5-dimethyl cyclohexanone (10): It was obtained as a creamish white solid; yield $31.73 \%$; m.p2 $18.5^{\circ} \mathrm{C}$; Anal. calc for $\mathrm{C}_{23} \mathrm{H}_{27} \mathrm{~N}_{3} \mathrm{O}$ C, 76.42 ; $\mathrm{H}, 7.53$; N,11.62; found: C, 76.40; H, 7.50; N, 11.61; IR (KBr) $v$ max: $\mathrm{cm}^{-1}$ : 3278, 3180, 2923, 1599, 1510, 1381 and $1339 \mathrm{~cm}^{-1} ; \mathrm{H}$ NMR $(400 \mathrm{MHz}$, DMSO): 0.99 (3H, d, J=17.2, $\left.\mathrm{CH}_{3}\right) ; 1.04\left(3 \mathrm{H}, \mathrm{d}, \mathrm{J}=14, \mathrm{CH}_{3}\right) ; 2.05(1 \mathrm{H}, \mathrm{d}$, $\left.\mathrm{J}=11.2, \mathrm{CH}_{2}\right) ; 2.18\left(1 \mathrm{H}, \mathrm{d}, \mathrm{J}=14.8, \mathrm{CH}_{2}\right) ; 2.51\left(2 \mathrm{H}, \mathrm{m}, \mathrm{CH}_{2}\right) ; 6.04(8 \mathrm{H}, \mathrm{m}$, Ar-H); ESI MS m/z: 360[M-1]

1-((E)-2,5-dihydro-2-(4-hydroxy-3-ethoxyphenyl)-1H-benzo[b][1,4] diazepin)-5-dimethyl cyclohexanone (11): It was obtained as a light yellow solid; yield $45.14 \%$; m.p-215.3 ${ }^{\circ} \mathrm{C}$; Anal. calc for $\mathrm{C}_{23} \mathrm{H}_{26} \mathrm{~N}_{2} \mathrm{O}_{3} \mathrm{C}$, 72.99; H, 6.92; N, 7.40; found: C, 72.97; H, 6.90; N, 7.38; IR ( $\mathrm{KBr}) v$ max: $\mathrm{cm}^{-1}: 3296,3238,2925,1599,1509,1390$ and $1121 \mathrm{~cm}^{-1} ;{ }^{1} \mathrm{H}$ NMR $\left(400 \mathrm{MHz}, \mathrm{CDCl}_{3}\right): 1.10\left(6 \mathrm{H}, \mathrm{m}, \mathrm{CH}_{3}\right) ; 1.37\left(5 \mathrm{H}, \mathrm{m}, \mathrm{OC}_{2} \mathrm{H}_{5}\right) ; 2.32(4 \mathrm{H}, \mathrm{m}$, $\left.\mathrm{CH}_{2}\right) ; 3.96(2 \mathrm{H}, \mathrm{m}, \mathrm{NH}) ; 5.39(1 \mathrm{H}, \mathrm{s}, \mathrm{CH}) ; 5.85(1 \mathrm{H}, \mathrm{s}, \mathrm{OH}) ; 6.54(8 \mathrm{H}, \mathrm{m}$, Ar-H); ESI MS m/z: $379[\mathrm{M}+1]$ 
1-((E)-2-(3,4-difluorophenyl) 2,5-dihydro-1H-benzo[b][1,4]diazepin)-5dimethylcyclohexanone (12): It was obtained as orange solid; yield 19.59\%; m.p-215.6 $6^{\circ}$; Anal. calc for $\mathrm{C}_{21} \mathrm{H}_{20} \mathrm{~F}_{2} \mathrm{~N}_{2} \mathrm{O}$ C, $71.17 ; \mathrm{H}, 5.69 ; \mathrm{N}, 7.90$; found: C, 71.16; H, 5.67; N, 7.88; IR (KBr) v max: $\mathrm{cm}^{-1}$ : 3298, 3187, 2929, 1582, 1515,1383 and $1276 \mathrm{~cm}^{-1} ;{ }^{1} \mathrm{H} \operatorname{NMR}\left(400 \mathrm{MHz}, \mathrm{CDCl}_{3}\right): 1.10\left(6 \mathrm{H}, \mathrm{m}_{2} \mathrm{CH}_{3}\right)$; $2.23 \quad\left(2 \mathrm{H}, \mathrm{q}, \mathrm{CH}_{2}\right) ; 2.45 \quad\left(1 \mathrm{H}, \mathrm{d}, \mathrm{J}=16, \mathrm{CH}_{2}\right) ; 2.59 \quad\left(1 \mathrm{H}, \mathrm{d}, \mathrm{J}=16, \mathrm{CH}_{2}\right) ; 5.89$ $(1 \mathrm{H}, \mathrm{s}, \mathrm{CH}) ; 6.44(7 \mathrm{H}, \mathrm{m}, \mathrm{Ar}-\mathrm{H})$;ESI MS m/z: 355[M+1]

1-((E)-2,5-dihydro-2-(3,4,5-trimethoxyphenyl-1H-benzo[b][1,4] diazepin)-5-dimethylcyclo hexanone (13): It was obtained as a white solid; yield 27.60\%;m.p-133.6 $6^{\circ}$; Anal. calc for $\mathrm{C}_{24} \mathrm{H}_{28} \mathrm{~N}_{2} \mathrm{O}_{4} \mathrm{C}$, 70.57; $\mathrm{H}, 6.91$; N, 6.86; found: C, 70.57; H, 6.91; N, 6.86; IR (KBr)v max: $\mathrm{cm}^{-1}: 3378$, 2925, 1590, 1534, 1383 and $1123 \mathrm{~cm}^{-1} ;{ }^{1} \mathrm{H}$ NMR (400MHz, $\left.\mathrm{CDCl}_{3}\right): 1.09$ $\left(3 \mathrm{H}, \mathrm{s}, \mathrm{CH}_{3}\right) ; 1.16\left(3 \mathrm{H}, \mathrm{s}, \mathrm{CH}_{3}\right) ; 2.24\left(4 \mathrm{H}, \mathrm{m}, \mathrm{CH}_{2}\right) ; 3.63\left(9 \mathrm{H}, \mathrm{s}, \mathrm{OCH}_{3}\right)$; $5.85(1 \mathrm{H}, \mathrm{s}, \mathrm{CH}) ; 6.30(7 \mathrm{H}, \mathrm{m}, \mathrm{Ar}-\mathrm{H})$; ESI MS m/z: 409 [M+1]

1-((E)-2,5-dihydro-2-p-tolyl-1H-benzo[b] [1,4]diazepin)-5-dimethyl cyclo hexanone (14): It was obtained as a light brown solid; yield 39.06\%; m.p209.4 ${ }^{\circ} \mathrm{C}$; Anal. calc for $\mathrm{C}_{22} \mathrm{H}_{24} \mathrm{~N}_{2} \mathrm{O}$ : C, 79.48; H, 7.28; N, 8.43; found: C, 79.46; H, 7.26; N, 8.41;IR (KBr) v max: $\mathrm{cm}^{-1}: 3306,3144,2922,1591,1537$ and $1377 \mathrm{~cm}^{-1} ;{ }^{1} \mathrm{H}$ NMR $\left(400 \mathrm{MHz}, \mathrm{CDCl}_{3}\right): 1.07\left(3 \mathrm{H}, \mathrm{s}, \mathrm{CH}_{3}\right) ; 1.14(3 \mathrm{H}$, $\left.\mathrm{s}, \mathrm{CH}_{3}\right) ; 2.30\left(4 \mathrm{H}, \mathrm{m}, \mathrm{CH}_{2}\right) ; 2.57\left(1 \mathrm{H}, \mathrm{d}, \mathrm{J}=15.6, \mathrm{CH}_{3}\right) ; 5.92(1 \mathrm{H}, \mathrm{s}, \mathrm{CH})$; $6.43(8 \mathrm{H}, \mathrm{m}, \mathrm{Ar}-\mathrm{H})$;ESI MS m/z: $333[\mathrm{M}+1]$

1-((E)-2-(2,4-dichlorophenyl)-2,5-dihydro-1H-benzo[b][1,4]diazepin)5-dimethylcyclohexanone (15): It was obtained as a creamish white solid; yield 34.16\%; m.p - $199.2^{\circ} \mathrm{C}$; Anal. calc for $\mathrm{C}_{21} \mathrm{H}_{20} \mathrm{Cl}_{2} \mathrm{~N}_{2} \mathrm{O}$ : C, 65.12; $\mathrm{H}$, 5.20 ; N, 7.23; found: C, 65.10; H, 5.18; N, 7.21; IR (KBr)v max: $\mathrm{cm}^{-1}$ : 3292, 3189, 2956, 1585, 1529, 1384 and $752 \mathrm{~cm}^{-1}$; ${ }^{1} \mathrm{H} \mathrm{NMR}(400 \mathrm{MHz}$, $\left.\mathrm{CDCl}_{3}\right): 1.14\left(3 \mathrm{H}, \mathrm{s}, \mathrm{CH}_{3}\right) ; 1.18\left(3 \mathrm{H}, \mathrm{s}, \mathrm{CH}_{3}\right) ; 2.23\left(4 \mathrm{H}, \mathrm{m}, \mathrm{CH}_{2}\right) ; 2.48$ $(1 \mathrm{H}, \mathrm{d}, \mathrm{J}=16, \mathrm{NH}) ; 2.63(1 \mathrm{H}, \mathrm{d}, \mathrm{J}=16, \mathrm{NH}) ; 4.91(1 \mathrm{H}, \mathrm{s}, \mathrm{CH}) ; 6.19(8 \mathrm{H}$, $\mathrm{m}, \mathrm{Ar}-\mathrm{H}) ; \mathrm{ESI} \mathrm{MS} \mathrm{m} / \mathrm{z}: 385$ [M-2]

1-((E)-2,5-dihydro-2-(4-isopropylphenyl)-1H-benzo[b][1,4]diazepin)5-dimethylcyclohexanone (16): It was obtained as a lemon yellow solid; yield 27.04\%; m.p-248.2 $2^{\circ}$; Anal. calc for $\mathrm{C}_{24} \mathrm{H}_{28} \mathrm{~N}_{2} \mathrm{O}$ : C, 79.96; $\mathrm{H}, 7.83$; $\mathrm{N}, 7.77$; found: C, 79.94; $\mathrm{H}, 7.81 ; \mathrm{N}, 7.76$; IR (KBr) $v$ max: $\mathrm{cm}^{-1}: 3311$, $3179,2958,1902,1782$ and $1332 \mathrm{~cm}^{-1}$; ${ }^{1} \mathrm{H}$ NMR (400MHz, $\left.\mathrm{CDCl}_{3}\right): 1.10$ $\left(6 \mathrm{H}, \mathrm{s}, \mathrm{CH}_{3}\right) ; 1.14\left(6 \mathrm{H}, \mathrm{m}\right.$, Isopropyl $\left.\mathrm{CH}_{3}\right) ; 2.23\left(4 \mathrm{H}, \mathrm{m}, \mathrm{CH}_{2}\right) ; 2.78(1 \mathrm{H}$, m, Isopropyl-CH); $5.92(1 \mathrm{H}, \mathrm{s}$, Chiral CH); $6.42(9 \mathrm{H}, \mathrm{m}, \mathrm{Ar}-\mathrm{H})$; ESI MS $\mathrm{m} / \mathrm{z}: 361[\mathrm{M}+1]$

1-((E)-2-(3-bromophenyl)-2,5dihydro-1H-benzo[b][1,4]diazepin)5-dimethyl cyclo hexanone (17): It was obtained as a brown solid; yield 35.58\%; m.p-223.7 C; Anal. calc for $\mathrm{C}_{21} \mathrm{H}_{21} \mathrm{BrN}_{2} \mathrm{O}$ C, 63.48; $\mathrm{H}, 5.33 ; \mathrm{N}$, 7.05; found:C, 63.46; H, 5.31; N, 7.03; IR (KBr) v max: $\mathrm{cm}^{-1}: 3318,3184$, 2955, 1586, 1503, 1384 and $751 \mathrm{~cm}^{-1} ;{ }^{1} \mathrm{H}$ NMR (400MHz, $\left.\mathrm{CDCl}_{3}\right): 1.10$ $\left(3 \mathrm{H}, \mathrm{s}, \mathrm{CH}_{3}\right) ; 1.16\left(3 \mathrm{H}, \mathrm{s}, \mathrm{CH}_{3}\right) ; 2.28\left(4 \mathrm{H}, \mathrm{m}, \mathrm{CH}_{2}\right) ; 5.90(1 \mathrm{H}, \mathrm{s}, \mathrm{CH}) ; 6.44$ (9H, m, Ar-H); ESI MS m/z: 396 [M-1]

1-((E)-2-(2-chloro phenyl) 2,5dihydro-1H-benzo[b][1,4] diazepin)5-dimethyl cyclo hexanone (18): It was obtained as a brown solid; yield 34.55\%; m.p-197.8 ${ }^{\circ} \mathrm{C}$; Anal. calc for $\mathrm{C}_{21} \mathrm{H}_{21} \mathrm{ClN}_{2} \mathrm{O}$ : C, 71.48; $\mathrm{H}, 6.00 ; \mathrm{N}$, 7.94; found:C, 71.48; H, 6.00; N, 7.94; IR (KBr)v max: $\mathrm{cm}^{-1}$ : 3337, 3186, 2931, 1586, 1508, 1381 and $750 \mathrm{~cm}^{-1} ;{ }^{1} \mathrm{H} \mathrm{NMR}\left(400 \mathrm{MHz}, \mathrm{CDCl}_{3}\right): 1.17$ $\left(6 \mathrm{H}, \mathrm{s}, \mathrm{CH}_{3}\right) ; 2.37\left(2 \mathrm{H}, \mathrm{m}, \mathrm{CH}_{2}\right) ; 2.47\left(1 \mathrm{H}, \mathrm{d}, \mathrm{J}=16, \mathrm{CH}_{2}\right) ; 2.62(1 \mathrm{H}, \mathrm{d}$, $\left.\mathrm{J}=15.2, \mathrm{CH}_{2}\right) ; 6.27(1 \mathrm{H}, \mathrm{s}, \mathrm{CH}) ; 6.44(9 \mathrm{H}, \mathrm{m}, \mathrm{Ar}-\mathrm{H})$; ESI MS m/z: 353 $[\mathrm{M}+1]$

\section{BIOLOGICAL ACTIVITY}

In-vitro Antitubercular Activity Screening - MABA assay

The test compounds were screened for preliminary anti-TB activity against pathogenic strains of M. tuberculosis $\mathrm{H}_{37} \mathrm{Rv}$ (ATCC 27294), using Microplate Alamar Blue assay (MABA) ${ }^{27}$ The anti-tubercular activity results were presented in Table 1.

\begin{tabular}{|c|c|c|c|c|c|}
\hline \multirow[t]{2}{*}{ S.No } & \multirow[t]{2}{*}{ Cpd code } & MABA Assay & \multirow[t]{2}{*}{ S.No } & \multirow[t]{2}{*}{ Cpd code } & \multirow{2}{*}{$\frac{\text { MABA Assay }}{\text { MIC }(\mu \mathrm{g} / \mathrm{ml})}$} \\
\hline & & MIC $(\mu \mathrm{g} / \mathrm{ml})$ & & & \\
\hline 1. & KR-01 & 12.5 & 12. & KR-12 & 25 \\
\hline 2. & KR-02 & 12.5 & 13. & KR-13 & 50 \\
\hline 3. & KR-03 & 25 & 14. & KR-14 & 50 \\
\hline 4. & KR-04 & 6.25 & 15. & KR-15 & 50 \\
\hline 5. & KR-05 & 100 & 16. & KR-16 & 25 \\
\hline 6. & KR-06 & 50 & 17. & KR-17 & 50 \\
\hline 7. & KR-07 & 25 & 18. & KR-18 & 50 \\
\hline 8. & KR-08 & 25 & 19. & Rifampicin & - \\
\hline 9. & KR-09 & 1.6 & 20. & Pyrazinamide & 3.125 \\
\hline 10. & KR-10 & 1.6 & 21. & Streptomycin & 6.25 \\
\hline 11. & KR-11 & 3.125 & 22. & Ciprofloxacin & 3.125 \\
\hline
\end{tabular}

\section{In vitro antimicrobial screening}

The antimicrobial susceptibility testing was performed in vitro by Agar well diffusion method. ${ }^{28}$ The test organisms used were: Gram-positive bacteria - Staphylococcus aureus (NCIM 2122), Gram-negative bacteriaEscherichia coli (NCIM 2137) and fungi- Candida albicans (NCIM 3102), Asperigillus niger (NCIM 652). All the compounds were tested at a dose level of $100 \mu \mathrm{g}(0.1 \mathrm{~mL})$ and DMSO was used as a control whereas Rifampicin and Ketoconazole were taken as standard drugs. None of the compounds had shown antibacterial and antifungal activity.

\section{RESULTS}

A total of eighteen 1, 4-Benzodiazepines derivatives were synthesized by using established protocols. The compounds were obtained in good yield and were characterized by using spectral analysis including NMR, IR and Mass. The aldehydes were selected in order to probe the role of bulkiness and nucleophilicity on anti-TB activity. The synthesized compounds were screened for antimicrobial and anti-tubercular activities. Among all, Compound 9, 10 (MIC $1.6 \mu \mathrm{g} / \mathrm{mL}$ ) followed by compound $\mathbf{1 1}(3.12 \mu \mathrm{g} / \mathrm{mL})$, compound $4(6.25 \mu \mathrm{g} / \mathrm{mL})$ had shown potent anti-TB activity. None of the compounds had shown antimicrobial activity at $100 \mu \mathrm{g} / \mathrm{mL}$, indicating selective anti-tubercular activity profile of these compounds.

\section{DISCUSSION}

New anti-TB drugs are urgently needed to battle drug-resistant Mycobacterium tuberculosis (MTB) strains and to shorten the long treatment regimen. The persistence of this disease has its roots in the resilience of mycobacterium to antibiotics/host-immunity, patient non-compliance and public ignorance. Steady raise in multidrug resistant infections and paltry presence of novel anti TB drugs in the pipeline is posing a grave threat to humanity. Anti TB drug discovery and development has several bottlenecks. For generations, TB remained a "poor people's" disease. Globally, TB patient care is largely managed by the respective governments, hence there is no assured financial gains. Secondly, clinical trials for TB drugs are hampered by ethical issues. Lastly, an antibiotic drug, when listed for TB therapy, loses its ground for treating other diseases. Financial and time constraints made TB drug research a non-productive task for many pharmaceutical companies. Focused on these issues, we are specifically interested to develop novel TB drugs with novel mechanism of action which ultimately impede the resistance problem. 
Antimycobacterial drug discovery poses unique challenges in terms of safety, efficacy and novelty. Anti TB drug treatment usually spans 3 months to one year. Hence long term exposure to anti TB drugs shall have influence on the microbial flora of gut and other parts of our body. Hence it is sought to discover a highly potent anti TB drug with high selectivity index. Heterocyclic molecules are known to possess wide range of clinically useful pharmacological activities. Benzodiazepine derivatives are well known for their anti-anxiety activity. This pharmacophore is also present in compounds showing clinically useful pharmacological activities. ${ }^{29}$ Ubiquitous presence of 1, 4-benzodiazepines in chemical literature ${ }^{29-30}$ is undoubtedly a consequence of the multifarious biological response which they elicit in combating a variety of body ailments.

This aroused our interest in the heterocycle of benzodiazepines and prompted us to undertake a study to seek structural modification of this nucleus to generate novel synthetic analogues of medicinal interest. Chemical literature is replete with examples showing that the incorporation of certain bioactive pharmacophore exerts a significant additive effect on the overall biological profile of the drug. Based on this trend, in the present work we prepared different aryl substituted 1, 4-benzodiazepines to obtain novel anti TB agents.

A total of eighteen 1,4-Benzodiazepines derivatives were synthesized by condensation of o-phenylenediamines with 1, 3-diketone (Dimedone). Further these synthesized derivatives were analyzed by IR, NMR and MASS spectral studies.

After the confirmation by spectral data, they were screened for antimicrobial and anti-tubercular activities. Among all, Compound 9, 10 (MIC $1.6 \mu \mathrm{g} / \mathrm{mL}$ ) followed by compound $\mathbf{1 1}(3.12 \mu \mathrm{g} / \mathrm{mL})$, compound 4 $(6.25 \mu \mathrm{g} / \mathrm{mL})$ had shown potent selective anti-TB activity. Selective anti $\mathrm{TB}$ is utmost important especially in the TB drug therapy. In order to check the selective anti TB activity, anti-microbial activity was performed and none of the compounds possessed anti-microbial activity.

\section{SAR of Anti TB Activity observed in case of 1 , 4-Benzodiazepines derivatives}

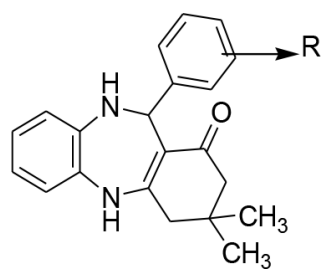

\section{Basic chemical moiety}

Anti-TB activity was observed without any substitution on the ring-R. Substitution of electron donating groups like $-\mathrm{OCH}_{3}$ at both para and meta positions on the ring- $\mathrm{R}$ increased anti-TB activity. Substitution of electron donating groups at meta position like $-\mathrm{OC}_{2} \mathrm{H}_{5}$ on the ring$\mathrm{R}$ slightly reduced anti-TB activity i.e., may be due to increase in alkyl chain. No anti-bacterial and anti-fungal activity with and without substitution on the ring- $\mathrm{R}$ was observed. Substitution of electron withdrawing groups on the ring- $\mathrm{R}$ at para position like $-\mathrm{F}$ increased anti-TB activity. Along with para position if $-\mathrm{F}$ also substituted on the ring- $\mathrm{R}$ at meta position reduced anti-TB activity. Substitution of electron withdrawing groups like $-\mathrm{Br},-\mathrm{Cl}$ at ortho, para and meta positions on the ring- $\mathrm{R}$ reduced anti-TB activity.

\section{CONCLUSION}

In conclusion, we synthesized eighteen 1, 4-Benzodiazepines derivatives. These compounds were screened for their anti-tubercular and antimicrobial activities. Among all, compounds 9, 10 (MIC $1.6 \mu \mathrm{g} / \mathrm{mL}$ ) followed by compound $\mathbf{1 1}(3.12 \mu \mathrm{g} / \mathrm{mL})$ and compound $4(6.25 \mu \mathrm{g} / \mathrm{mL})$ had shown anti-tubercular activity against $M$. tuberculosis $\mathrm{H} 37 \mathrm{Rv}$ which are comparable with the standard drugs. None of the compounds had shown antibacterial and antifungal activity.

\section{ACKNOWLEDGEMENT}

M.M.K and T. Mohan financially supported from DST-SERB EMR/2014/000289, New Delhi, India and G.P.V.S partially supported from (UGC-NF), F./2014-15/NFO-2014-15-OBC-AND-18673/(SA-III/ Website) New Delhi, India.

\section{CONFLICT OF INTEREST}

The authors declare no conflict of interest.

\section{ABBREVIATIONS}

MDR: Mutliple drug resistance; XDR: Extensively drug resistance; TB: Tuberculosis; MABA: Microplate alamar blue assay; DMSO: Dimethy sulphoxide; TLC: Thin layer chromatography; RBF: Round bottom flask.

\section{SUMMARY}

A novel method was reported for the synthesis of of 1,4 benzodiazepine derivatives. Dimedone usage provoked novelty in the scheme. Among the synthesized compounds,compound 4,9,10,11 had shown potent and selective anti TB activity.

\section{REFERENCES}

1. Sheela S, Friedland G. Extensively drug-resistant tuberculosis: A new face to an old pathogen. Annu Rev Med. 2009;60:307-20.

2. Website information- WHO Global TB Report 2015- TB Facts. TB report.org [homepage on internet]. $20^{\text {th }}$ edidtion world health organization. Available from [http://www.who.int/tb/publications/global_report/en/]

3. Parida SK, Axelsson-Robertson R, Rao MV, Singh N, Master I, Lutckii $A$, et al. Totally drug-resistant tuberculosis and adjunct therapies. J Intern Med. 2015;277(4):388-405.

4. Udwadia ZF, Amale RA, Ajbani KK, Rodrigues C. Total drug-resistant tuberculosis in India. Clin Infect Dis. 2012;54(4):579-81.

5. Brigden G, Hewison C, Varaine F. New developments in the treatment of drugresistant tuberculosis: Clinical utility of bedaquiline and delamanid. Infect. Drug Resist. 2015;8:367-78.

6. Landquist JK, Katritzky AR, Rees CW. In Comprehensive Heterocyclic Chemistry. 1984; $1: 166$.

7. Sternbach LH, Angew Chem. 1,4-benzodiazepines. Chemistry and some aspects of the structure-activity relationship. Int Ed Engl. 1971;10(1):34-43

8. Randall LO. Metabolism of adenine and derivatives by cerebral tissues, superfused and electrically stimulated. Psychopharmacological Agents. 1974;3:175-281.

9. Hsu MC, Schutt AD, Holly M, Slice LW, Sherman MI, Richman DD, et al. Advances in virus research. Science. 1991;254(5039):1799-802.

10. Paumels R, Andries K, Desmyter J, Schols D, Kukla MJ, Breslin H, et al. Potent and selective inhibition of HIV-1 replication in vitro by a novel series of TIBO derivatives. Nature. 1990;343(6257):470-4.

11. Olkkola KT, Ahonen J. Midazolam and other benzodiazepines. Hand Book Exp Pharmacol. 2008;182:335-60.

12. Kamal A, Shankaraiah N, Prabhakar S, Reddy CR, Markandeya N, Laxma K, et al. Solid-phase synthesis of new pyrrolobenzodiazepine-chalcone conjugates: DNA-binding affinity and anticancer activity. Bioorg Med Chem Lett. 2008; 18(7):2434-9.

13. Stahlofen P, RiedW. Cyclocondensation of 1,2 diamines Chem Ber. 1957;90:815-9.

14. ReidW, Torinus E. 1,5 benzodiazepenes and 1,5 benzodiazepinium salts. Chem Ber. 1959;92:2902-8.

15. Herbert JA, Suschitzky H. Syntheses of heterocyclic compounds. Substituted 2,3-dihydro-1H-1,5-benzodiazepines. J Chem Soc Perkin Trans. 1974;1:2657-61.

16. Morales HR, Bulbarela A, Contreras R. New synthesis of dihydro- and tetrahydro-1,5- benzodiazepines by reductive condensation of o-phenylenediamine and ketones in the presence of sodium borohydride. Heterocycles. 1986;17(39):135-9.

17. Jung DI, Choi TW, Tim YY, Kim IS, Park YM, Lee YG, et al. Synthesis of. 1,5-benzodiazepine derivatives. Synth Commun. 1999;30(32):1941-51.

18. Shi RX, Liu YK, Xu ZY. Sodium tetrachloroaurate(III) dihydrate-catalyzed efficient synthesis of 1,5-benzodiazepine and quinoxaline derivatives. J Zhejiang Univ 
Sci B. 2010;11(2):102-8.

19. Sajjadifar $\mathrm{S}$, Vahedi $\mathrm{H}$, Massoudi $\mathrm{AH}$, Louie O. New $3 \mathrm{H}$-Indole Synthesis by Fischer's Method. Molecules. 2010;15(4):2491-8.

20. Zolfigol MA, Vahedi H, Massoudi AH, Sajjadifar S, Louie O, Javaherneshan N. Mild and efficient one pot synthesis of benzoimidazoles from aldehyde by using BSA a new catalyst. Clinical Biochemistry. 2011;44(13):219-22.

21. Zolfigol MA, Khazaei A, Vahedi H, Mokhlesi M, Sajjadifar S, Pirveysian M. Heterogeneous and Catalytic Thiocyanation of Aromatic Compounds in Aqueous Media. Phosphorus, Sulfur, and Silicon and the Related Elements. 2012;187(3):295-304.

22. Sajjadifar S, Mirshokraie SA, Javaherneshan N, Louie O. SBSA as a New and Efficient Catalyst for the One-Pot Green Synthesis of Benzimidazole Derivatives at Room Temperature. American Journal of Organic Csshemistry. 2012;2(2):1-6.

23. Sajjadifar S. Green Thiocyanation of Aromatic and Heteroaromatic Compounds by Using Silica Boron Sulfonic Acid as a New Catalyst and H2O2. Journal of Organic Chemistry. 2012;2(5):116-21.

24. Sajjadifar S, Louie O. Regioselective Thiocyanation of Aromatic and Heteroaro- matic Compounds by Using Boron Sulfonic Acid as a New, Efficient, and Cheap Catalyst in Water. Journal of Chemistry. 2013;10:6

25. Sajjadifar S. Boron Sulfonic Acid (2008-2012). International Journal of Chem Tech Research. 2013;5:385-9.

26. Jarikote DV, Siddiqui SA, Rajagopal R, Thomas D, Lahoti RJ, Srinivasan KV. Room temperature ionic liquid promoted synthesis of 1,5-benzodiazepine derivatives under ambient conditions. Tetrahedron Lett. 2003;44(9):1835-8.

27. Lourenco CS, Desouza M, et al. Evaluation of anti-tubercular activity of nicotinic and isoniazid analogues. ARKIVOC. 2007;15:181-91.

28. Mounyr B, Moulay S, Saad K. Methods for in vitro evaluating antimicrobial activity: A review. J Pharm Anal. 2016;6(2):71-9.

29. Patel NB, Rathod RD. Synthesis and antimicrobial studies of novel biological heterocycles. Chin J Chem. 2008;26(12):2233-40.

30. Dallemagne P, Khanh LP, Alsai A, Varlet I, Collot V, Paillet M, et al. Synthesis and biological evaluation of five-membered heterocycles fused to cyclopenta thiophene as new antitumor agents. Bioorg Med Chem. 2003;11(7):1161-70.

Article History: Submission Date : 14-02-2018; Revised Date : 23-05-2018; Acceptance Date : 08-06-2018.

Cite this article: Kumar MMK, Mohan T, mai GK, Sangeeta GPV, Nagasree KP. Synthesis, Characterization and Biological Evaluation of Novel 1, 4-Benzodiazepine Derivatives as Potent Anti-Tubercular Agents. J Young Pharm. 2018;10(3):267-71. 